\title{
Universal transduction scheme for nanomechanical systems based on dielectric forces
}

\author{
Quirin P. Unterreithmeier ${ }^{1}$, Eva M. Weig ${ }^{1}$ \& Jörg P. Kotthaus ${ }^{1}$
}

\begin{abstract}
Any polarizable body placed in an inhomogeneous electric field experiences a dielectric force. This phenomenon is well known from the macroscopic world: a water jet is deflected when approached by a charged object. This fundamental mechanism is exploited in a variety of contexts-for example, trapping microscopic particles in an optical tweezer ${ }^{1}$, where the trapping force is controlled via the intensity of a laser beam, or dielectrophoresis ${ }^{2}$, where electric fields are used to manipulate particles in liquids. Here we extend the underlying concept to the rapidly evolving field of nanoelectromechanical systems ${ }^{3,4}$ (NEMS). A broad range of possible applications are anticipated for these systems $s^{5,6,7}$, but drive and detection schemes for nanomechanical motion still need to be optimized ${ }^{8,9}$. Our approach is based on the application of dielectric gradient forces for the controlled and local transduction of NEMS. Using a set of on-chip electrodes to create an electric field gradient, we polarize a dielectric resonator and subject it to an attractive force that can be modulated at high frequencies. This universal actuation scheme is efficient, broadband and scalable. It also separates the driving scheme from the driven mechanical element, allowing for arbitrary polarizable materials and thus potentially ultralow dissipation NEMS ${ }^{10}$. In addition, it enables simple voltage tuning of the mechanical resonance over a wide frequency range, because the dielectric force depends strongly on the resonator-electrode separation. We use the modulation of the resonance frequency to demonstrate parametric actuation ${ }^{11,12}$. Moreover, we reverse the actuation principle to realize dielectric detection, thus allowing universal transduction of NEMS. We expect this combination to be useful both in the study of fundamental principles and in applications such as signal processing and sensing.
\end{abstract}

Common actuation mechanisms of nanomechanical resonators can be divided into local on-chip schemes and schemes relying on external excitation. The former are based on voltage-induced forces such as internal piezo-electrical ${ }^{9,12}$, capacitive ${ }^{11}$, magnetomotive ${ }^{13}$, electrothermal ${ }^{14}$ or static dipole-based dielectric ${ }^{15}$. Although highly integrable and efficient, these schemes impose constraints on material choice and geometry and thus mostly suffer from large dissipation $^{16}$. The latter employ external actuation such as photothermal ${ }^{17}$ or inertia-based piezo-actuated schemes ${ }^{10}$, which is less restrictive on system choice and hence advantageous in terms of dissipation ${ }^{3,10}$. However, attaining high-frequency actuation as well as integrability remains a challenge.

Here, we introduce a driving scheme that integrates external, yet local actuation for arbitrary resonators, directly based on electrical signals. It enables independent optimization of both the actuation and the resonant element. Our mechanism relies solely on dielectric interaction: A polarizable material experiences an attractive force in an inhomogeneous electric field directed towards the maximum field strength. In our case the polarizable element is a doubly clamped silicon nitride beam, as depicted in Fig. 1a, which serves as a lowdissipation radio-frequency (r.f.) resonator ${ }^{10}$. The inhomogeneous field in the beam plane is created by two subjacent gold electrodes (see inset of Fig. 1b). A static voltage $V_{\text {d.c. }}$ (direct current, d.c.) applied to the electrodes induces a strong dipolar moment in the resonator that in turn experiences an attractive force directed towards the electrodes. Modulating $V_{\text {d.c. }}$ with an r.f. signal $V_{\text {r.f. gives }}$ rise to an oscillating force component that drives the resonator perpendicularly to the chip plane.

To obtain quantitative insight into the dielectric forces, we carried out finite element simulations for the given geometry (see Fig. 1). The black line in Fig. $1 \mathrm{~b}$ depicts the dielectric force acting on the resonator as a function of its distance $d$ from the substrate. The force exhibits a maximum at a distance that is comparable, though somewhat smaller than our resonator-substrate separation of $d \approx 300 \mathrm{~nm}$. In addition, the simulations can be used to extract information on the underlying circuitry. The mutual capacitance of the electrodes is $C_{\text {mutual }} \approx 1.5 \mathrm{fF}$. Along with an impedance of $R \approx 50 \Omega$, this yields a cut-off frequency $f_{c}=1 /\left(2 \pi R C_{\text {mutual }}\right)$ in the terahertz regime, which goes well beyond attainable frequencies for driven nanomechanical systems ${ }^{4}$. A simple analytical model reproduces the simulated behaviour. As the electric field lines in the inset of Fig. 1b show, the overall dominant field component in the vicinity of the resonator is parallel to the surface
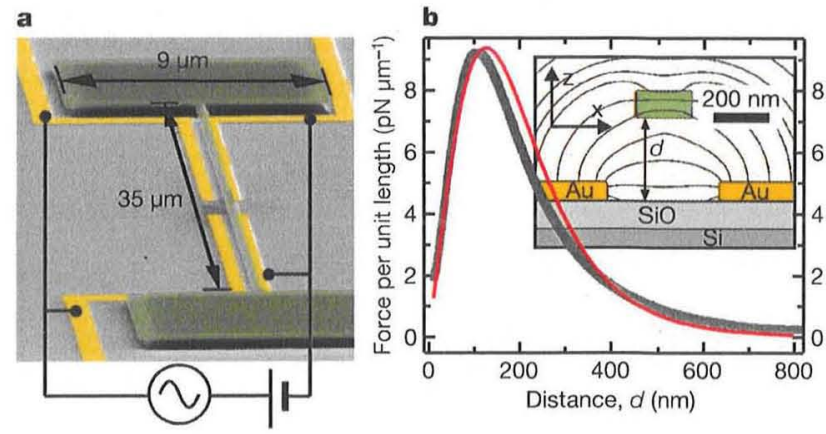

Figure 1 | Sample geometry and force acting on the nanomechanical resonator. a, Scanning electron micrograph of a representative device. The high-stress silicon nitride film (green) forms the suspended doubly clamped beam and its supports. The four nearby gold electrodes (yellow) are connected to both a d.c. and an r.f. voltage source used to polarize and resonantly excite the beam. $\mathbf{b}$, Electrostatic force per unit length in the $z$ direction, perpendicular to the sample plane, versus distance $d$ from the electrodes for $V_{\text {d.c. }}=2 \mathrm{~V}$ simulated by a finite element calculation (black) and approximated by an analytical fit (red). In our experiments $d$ is about $300 \mathrm{~nm}$. The inset depicts a cross-section of the device and shows the electric field lines obtained by the simulation. We note that the field component $E_{2}$ changes sign across the beam along the $x$ direction, giving rise to a finite $\partial E_{z} / \partial x$, as in equation (1). 
( $x$ direction). Therefore, the induced charge distribution on the resonator can be approximated by a dipole oriented in the $x$ direction proportional to the electric field component in this direction: $p_{x}=\chi E_{x}$, with susceptibility $\chi$. The charging $q_{i}$ of each electrode is described by a point charge. Neglecting the electrostatic contribution of the influenced charges, the $z$ component of the resulting force $F_{z}$ in this simple dipole approximation is proportional to the field gradient along the $x$ direction:

$$
\begin{aligned}
& F_{z}=p_{x} \frac{\partial E_{z}}{\partial x} \propto E_{x} E_{z} \\
& \text { with } \mathbf{E}(\mathbf{r})=\sum_{\mathrm{i}=1,2} q_{i} \frac{\mathbf{r}-\mathbf{r}_{i}}{\left|\mathbf{r}-\mathbf{r}_{i}\right|^{3}}
\end{aligned}
$$

Using the mutual distance of the electrodes $\left|\mathbf{r}_{1}-\mathbf{r}_{2}\right|$ and the resonator susceptibility $\chi$ as fit parameters, the simulated results are well approximated (see red line in Fig. 1b). Neglecting small deformations of the resonant element by electrical forces, equation (1) predicts a quadratic dependence on electric field, just as in the case of capacitive actuation". Weakly modulating the applied bias voltage therefore gives rise to an oscillating force:

$$
F\left[V_{\text {d.c. }}+V_{\text {r.f. }}\right]=c_{1}\left(V_{\text {d.c. }}+V_{\text {r.f. }}\right)^{2} \approx c_{1} V_{\text {d.c. }}^{2}+2 c_{1} V_{\text {d.c. }} V_{\text {r.f. }}
$$

with $c_{1}$ a constant

Equation (2) shows that two independent parameters ensure optimized actuation: while $V_{\text {r.f. }}$ is employed to actuate the oscillatory motion of the resonator, the amplitude of $V_{\text {d.c. }}$ independently controls the strength of the polarization. This striking behaviour is a distinct feature of electrical realizations of dielectric force gradients. Optically generated gradient forces which have recently been reported as actuation for nanomechanical resonators ${ }^{18}$ do not incorporate this polarization tunability because both polarization and actuating force result from the same laser field. Unlike for the related concept of laser tweezers employing polarizing quasi-static electrical fields ${ }^{1}$, the polarizing d.c. voltage allows efficient operation even in the case of a reduced susceptibility $\chi(\omega)$ in the frequency regime of resonator eigenmodes.

Our experiments are performed at room temperature in a vacuum of $P<3 \times 10^{-3}$ mbar to exclude gas damping. Resonators with typical dimensions of $(30-40) \times 0.2 \times 0.1 \mu \mathrm{m}^{3}$ (length $\times$ width $\times$ height) are fabricated from high-stress silicon nitride ${ }^{10}$ using standard lithographic methods. The drive electrodes are defined by lithographic postprocessing on fully released beams, enabled by the strong tensile stress of 1.4 GPa of the silicon nitride film. Several resonators processed on different sample chips were investigated. The results shown in this work are representative and have been taken from three distinct resonators.

Using a standard fibre-based optical interferometer ${ }^{19}$, we detect the out-of-plane displacement of the resonator sensitively enough to resolve the Brownian motion of the resonator, as shown in Fig. 2a. The fundamental resonance is described by a harmonic differential equation, with effective mass $m$, spring constant $k_{0}$, eigenfrequency $f_{0}=\sqrt{k_{0} / m} / 2 \pi$, mechanical quality factor $Q$ and external force $F$. For the investigated resonators, $f_{0}$ lies between 5 and $9 \mathrm{MHz}$, while $Q$ ranges from 100,000 to 150,000 , comparable to values reported elsewhere ${ }^{10}$. The frequency spectrum of the thermally driven system is Lorentzian. Its calculated amplitude ${ }^{20}$ is used as a calibration to convert the measured optical signal into displacement. Figure $2 \mathrm{~b}$ displays the driven resonator amplitude versus frequency along with a Lorentzian fit. The measured resonance amplitude (all indicated amplitudes are half-peak-to-peak amplitudes) for an actuation with $V_{\text {d.c. }}+V_{\text {r.t. }}=1 \mathrm{~V} \pm 0.2 \mathrm{mV}$ is about $\pm 0.8 \mathrm{~nm}$. A simple model based on the simulated forces yields $\pm 0.3 \mathrm{~nm}$ when assuming a dielectric constant of silicon nitride of 7 (the literature ${ }^{21}$ reports values between 6 and 9), which is in fair agreement. From the experimental data we estimate that a minimal actuation voltage $V_{\mathrm{r}, \mathrm{f} .}= \pm 5 \mu \mathrm{V}$ is sufficient to drive more strongly than the Brownian motion for a bandwidth of $50 \mathrm{~Hz}$. With the simulated value of $C_{\text {mutual }} \approx 1.5 \mathrm{fF}$ this translates into resonantly charging the electrodes by just 0.05 electrons, which

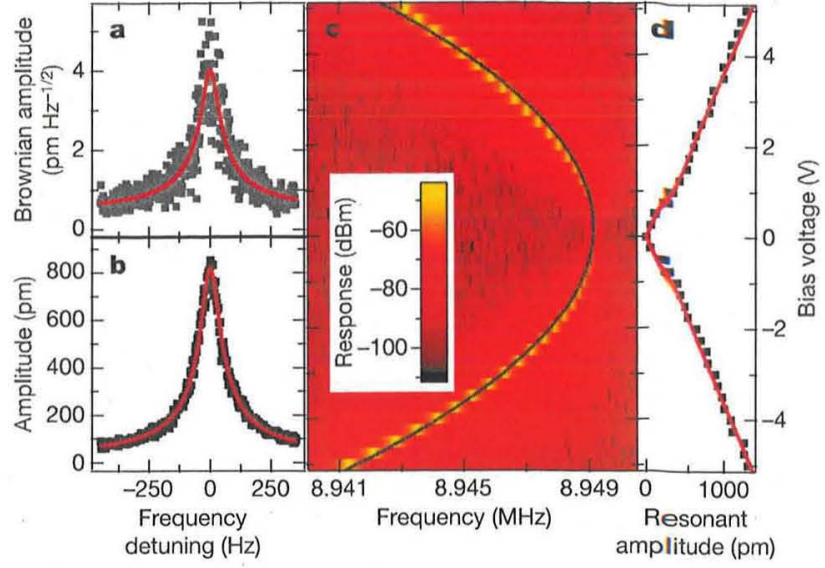

Figure 2 | Response of the dielectrically driven nanomechanical resonator. a, Brownian motion at room temperature for $V_{\text {d.c. }}=1 \mathrm{~V}$ without r.f. excitation. b, Dielectrically driven oscillation with $V_{\text {d.c. }}+V_{\text {r.f. }}=1 \mathrm{~V} \pm 0.2 \mathrm{mV}$, corresponding to an r.f. power of $-70 \mathrm{dBm}$. The data in $\mathbf{a}$ and $\mathbf{b}$ (dots) are well fitted by Lorentzians (red lines). The magnitude of the Brownian motion is used to convert the measured signal into the amplitude of the resonator displacement. $\mathbf{c}$ and $\mathbf{d}$, Response of the resonator as a function of frequency and d.c. bias voltage at r.f. drive $V_{\text {r.f. }}= \pm 0.06 \mathrm{mV}$. In c, the power response is logarithmically colour-coded. The resonance frequency decreases quadratically with $V_{\text {d.c. }}$ (fit shown by the black line). The resonant amplitude of $\mathbf{c}$ is displayed as a function of the d.c. bias in $\mathbf{d}$, reflecting the linear dependence of the resonator polarization on d.c. bias voltage (fit shown by the red line).

is below recently reported results'. In Fig. 2c individual power response traces are plotted as a function of frequency on a colour scale for a series of $V_{\text {d.c. }}$ and $V_{\text {r.f. }}= \pm 63 \mu \mathrm{V}$. The corresponding resonant amplitude is depicted in Fig. 2 d. It clearly scales linearly with the applied d.c. bias voltage, as expected from equation (2). The resonance frequency decreases quadratically with bias voltage (see fit indicated by solid black line in Fig. 2c). This can be readily understood from the force dependence on the distance $d$ (see Fig. 1b). Expanding this dependence around the equilibrium position $d_{0}$ yields:

$$
F\left[d_{0}+\delta d\right]=F_{0}+\frac{\partial F}{\partial d} \delta d+O(\delta d)^{2}
$$

The constant term $F_{0}$ leads to a new equilibrium position and can be ignored. However, the term linear in displacement (at the same time quadratic in applied voltage) acts as an additional spring constant on the resonator. It follows from Fig. $1 \mathrm{~b}$ that this contribution is negative for the given $d \approx 300 \mathrm{~nm}$. The resulting eigenfrequency $\tilde{f}_{0}$ therefore shifts in leading order with the observed quadratic voltage dependence:

$$
\tilde{f}_{0}=\frac{1}{2 \pi} \sqrt{\frac{k_{0}-c_{2} V_{\mathrm{d} . \mathrm{c}}^{2}}{m}} \approx f_{0}\left(1-\frac{c_{2} V_{\mathrm{d} . \mathrm{c} .}^{2}}{2 k_{0}}\right)
$$

with $c_{2}$ a constant

Figure 3a exhibits a frequency tuning range of more than $100 \mathrm{kHz}$, corresponding to approximately 1,000 full width at half maximum $(\mathrm{FWHM} \approx 100 \mathrm{~Hz}$ ).

Subject to strong actuation, the resonator response enters the nonlinear regime. This can be achieved for relatively small actuation powers, which do not give rise to a significant thermal heating of the sample (see Supplementary Information). Higher-order terms in displacement display similar tuning effects ${ }^{22}$, which will be presented elsewhere. The voltage tuning enables parametric excitation: a modulation of the resonance frequency at about $2 \tilde{f}_{0}$ can give rise to instability and self-oscillation of the system even without the applied resonant force $F^{23,24}$. Figure $3 \mathrm{~b}$ depicts the power response versus detection frequency $f$ near $\tilde{f}_{0}$ and r.f. frequency modulation power leading to the modulation amplitude $\delta f$ (see Fig. 3a). The characteristic Arnold 

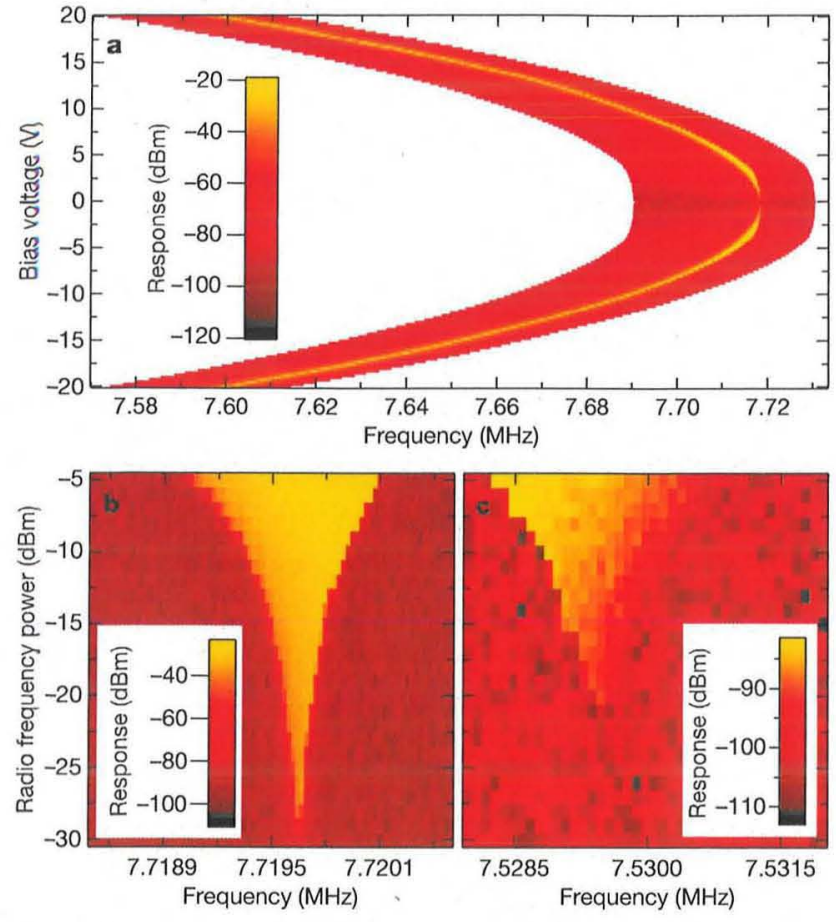

Figure 3 | Tuning and parametric transduction of the

nanoelectromechanical resonator. a, Power response of the resonator at $V_{\text {r.f. }}= \pm 2 \mathrm{mV}$ sampled around the resonance. To minimize long-term drifts traces are taken with increasing absolute value of d.c. bias reversing sign between consecutive traces (see Supplementary Information). b and c, Interferometric versus dielectric detection. b, Interferometrically measured power response subject to parametric actuation around $2 \tilde{f}_{0}$ at $V_{\text {d.c. }}=3 \mathrm{~V}$. The resonance frequency is modulated at $2 f$ with the r.f. power plotted on the $y$ axis, while the detection frequency $f$ is plotted along the $x$ axis. c, Power response of a parametrically excited resonator using dielectric detection at $V_{\text {d.c. }}=20 \mathrm{~V}$. As in $\mathbf{b}$, the resonator was driven by a frequency modulation at twice the detection frequency as a function of modulation power and detection frequency. The response reproduces the interferometrically measured data from b, even though a sample with a different electrical environment was used.

tongue ${ }^{7}$ indicates the region of instability and self-oscillation as experimental evidence of parametric actuation. In particular, when the resonance frequency $\tilde{f}_{0}$ is modulated at exactly twice its value with $\delta f(t)=\delta f \times \cos \left(2\left(2 \pi \tilde{f}_{0}\right) t\right)$, theory predicts the transition to occur when $\delta f>\tilde{f}_{0} / Q$. For the case shown in Fig. 3a, the transition is expected for a driving power of $-25 \mathrm{dBm}$, which is in good agreement with the data. However, we note that there is some ambiguity in defining the onset of spontaneous oscillation".

Reversing the actuation principle, we can also electrically detect the motion of the resonator locally. Therefore, on a different sample, a second pair of biased electrodes is introduced, which had previously been shunted with the driving electrodes (see Fig. 1a). The oscillating motion of the polarized resonator modulates the mutual capacitance of these electrodes, thereby creating an electrical signal. To avoid crosstalk from a resonant drive signal, the beam was parametrically excited around $2 \tilde{f}_{0}$, as discussed above. The dielectric detection scheme uses an impedance converter near the sample and is demonstrated in Fig. 3c. To estimate the achieved sensitivity, the response amplitudes of Fig. $3 \mathrm{~b}$ and $\mathrm{c}$ are compared when the resonator is driven $10 \mathrm{~dB}$ beyond the onset of spontaneous oscillation. An amplitude of $\pm 10 \mathrm{~nm}$ results in an electrical signal power of approximately $-80 \mathrm{dBm}$. As the noise level is about $-100 \mathrm{dBm}$ when measuring at $50 \mathrm{~Hz}$ bandwidth, the sensitivity is approximately $20 \mathrm{pm} \mathrm{Hz}^{-1 / 2}$ for the unoptimized device. An estimate of the limits of this detection scheme using a more advanced set-up can be found in the Supplementary Information.
Although other electrical displacement sensors have obtained higher sensitivities ${ }^{13,25,26}$, the integration with a highly efficient, material independent drive makes our dielectric scheme an interesting candidate for nanomechanical transduction.

In conclusion, by taking advantage of dielectric gradient forces, we realize and quantitatively validate a new and widely applicable actuation and readout scheme for nanoelectromechanical systems. It is on-chip and scalable to large arrays, broadband potentially beyo nd the gigahertz regime, and imposes no restrictions on the choice of resonator material. It thus enables the optimization of mechanical quality factors of the resonator without being bound by specific material requirements. The sensitivity of mechanical sensors scales with the quality factor ${ }^{3}$, so we anticipate the scheme to be of interest in the fast-developing field of sensing ${ }^{5,6}$. Capable of locally addressing individual resonators, it is particularly relevant for bio-sensing, where large arrays of individually addressable resonators are desirable to analyse multiple constituents Because the driven mechanical element can be fabricated separately from the actuating capacitor, it will also permit bottom-up fabrication $^{27}$. Using this actuation scheme we demonstrate strong electrical field-effect tuning of both the resonance amplitude and frequency. This facilitates parametric excitation of the resonator at $2 f$, thus allowing decoupled detection of its oscillation at $f$. The large frequency tuning range can, for example, be used for in-situ tuning of several mechanical elements into resonance ${ }^{28}$ or coupling to external elements ${ }^{29}$. Moreover, the combination of parametric excitation and (even weak) signal extraction enables digital signal processing based on mechanical elements, as has recently been demonstrated for microelectromechanical resonators ${ }^{12}$. With additional tuning, an almost ideal electromechanical bandpass filter has been suggested ${ }^{7}$. Whereas we already achieve highly efficient actuation, as reflected by the low driving voltages in the microvolt regime, the sensitivity of our detection scheme can be significantly enhanced by, for example, using a microwave tank circuit ${ }^{26}$. This also opens a pathway to cooling the mechanical eigenmodes ${ }^{26,30}$.

1. Ashkin, A. Optical trapping and manipulation of neutral particles using lasers. Proc. Natl Acad. Sci. USA 94, 4853-4860 (1997)

2. Jones, T. B. Electromechanics of Particles (Cambridge Univ. Press, 1995)

3. Ekinci, K. L. \& Roukes, M. L. Nanoelectromechanical systems. Rev. Sci. Instrum. 76, 061101 (2005)

4. Huang, X. M., Zorman, C. A., Mehregany, M. \& Roukes, M. L. Nanoelectromechanical systems: Nanodevice motion at microwave frequencies. Nature 421, 496 (2003)

5. Li, M. Tang H X \& Roukes, M. L Ultra-sensitive NEMS-based cantilevers for sensing, scanned probe and very high-frequency applications. Nature Nanotechnol. 2, 114-120 (2007)

6. Jensen, K., Kim, K. \& Zettl, A. An atomic-resolution nanomechanical mass sensor. Nature Nanotechnol. 3, 533-537 (2008).

7. Rhoads, J. F., Shaw, S. W., Turner, K. L. \& Baskaran, R. Tunable microelectromechanical filters that exploit parametric resonance. J. Vib. Acoust $120,423-430$ (2005)

8. Ekinci, K. L. Electromechanical transducers at the nanoscale: actuation and sensing of motion in nanoelectromechanical systems (NEMS). Small 1, 786-797 (2005).

9. Masmanidis, S. C. et al. Multifunctional nanomechanical systems via tunably coupled piezoelectric actuation. Science 317, 780-783 (2007).

10. Verbridge, S. S. Parpia, J. M. Reichenbach, R. B., Bellan, L. M. \& Craighead, H. G. High quality factor resonance at room temperature with nanostrings under high tensile stress. J. Appl. Phys. 99, 124304 (2006).

11. Rugar, D.\& Gruetter, P. Mechanical parametric amplification and thermomechanical noise squeezing. Phys. Rev. Lett. 67, 699-702 (1991).

12. Mahboob, I. \& Yamaguchi, H. Bit storage and bit flip operations in an electromechanical oscillator. Nature Nanotechnol. 3, 275-279 (2008).

13. Knobel, R. G. \& Cleland, A. N. Nanometre-scale displacement sensing using a single electron transistor. Nature 424, 291-293 (2003).

14. Bargatin, I., Kozinsky, I. \& Roukes, M. L. Efficient electrothermal actuation of multiple modes of high-frequency nanoelectromechanical resonators. Appl. Phys. Lett. 90, 093116 (2007)

15. Tang, H. X. Huang X.M. H. Roukes, M. L., Bichler, M. \& Wegscheider, W. Twodimensional electron-gas actuation and transduction for $\mathrm{GaAs}$ nanoelectromechanical systems. Appl. Phys. Lett. 81, 3879-3881 (2002).

16. Sekaric, L. Carr, D. W. Evoy, S., Parpia, J. M. \& Craighead, H. G. Nanomechanical resonant structures in silicon nitride: fabrication, operation and dissipation issues. Sens. Actuat. A 101, 215-219 (2002) 
17. Sampathkumar, A., Murray, T. W. \& Ekinci, K. L. Photothermal operation of high frequency nanoelectromechanical systems. Appl. Phys. Lett. 88, 223104 (2006).

18. Li, M. et al. Harnessing optical forces in integrated photonic circuits. Nature 456, 480-484 (2008)

19. Azak, N. O. et al. Nanomechanical displacement detection using fiber-optic interferometry. Appl. Phys. Lett. 91, 093112 (2007)

20. Gillespie, D. T. The mathematics of Brownian motion and Johnson noise. Am. J. Phys. 64, 225-240 (1996)

21. Gad-el-Hak, M. The MEMS Handbook 15-157 (CRC Press, 2001).

22. Zhang, W., Baskaran, R. \& Turner, K. Tuning the dynamic behavior of parametric resonance in a micromechanical oscillator. Appl. Phys. Lett. 82, 130-132 (2003).

23. Nayfeh, A. H. \& Mook, D. T. Nonlinear Oscillations Ch. 5 (Wiley, 1995).

24. Lifshitz, R. \& Cross, M. C. Response of parametrically driven nonlinear coupled oscillators with application to micromechanical and nanomechanical resonator arrays. Phys. Rev. B 67, 134302 (2003).

25. LaHaye, M. D., Buu, O., Camarota, B. \& Schwab, K. C. Approaching the quantum limit of a nanomechanical resonator. Science 304, 74-77 (2004).

26. Regal, C. A., Teufel, J. D. \& Lehnert; K. W. Measuring nanomechanical motion with a microwave cavity interferometer. Nature Phys. 4, 555-560 (2008).

27. $\mathrm{Li}, \mathrm{M}$. et al. Bottom-up assembly of large-area nanowire resonator arrays. Nature Nanotechnol. 3, 88-92 (2008).
28. Spletzer, M., Raman, A., Wu, A. Q., Xu, X. \& Reifenberger, R. Ultrasensitive mass sensing using mode localization in coupled microcantilevers. Appl. Phys. Lett. 88, 254102 (2006).

29. Cleland, A. N.\& Geller, M. R. Superconducting qubit storage and entanglement with nanomechanical resonators. Phys. Rev. Lett. 93, 070501 (2004).

30. Brown, K. R. et al. Passive cooling of a micromechanical oscillator with a resonant electric circuit. Phys. Rev. Lett. 99, 137205 (2007).

Acknowledgements Financial support by the Deutsche Forschungsgemeinschaft via project Ko 416/18, the German Excellence Initiative via the Nanosystems Initiative Munich (NIM) and LMUexcellent as well as LMUinnovativ is gratefully acknowledged.

Author Contributions The experiment was performed and analysed by Q.P.U.; the results were discussed and the manuscript was written by all authors. 